\title{
Prevalência de lesões nos ombros de docentes da rede de ensino público da cidade de Montes Claros - MG
}

\author{
Prevalence of injuries in teachers 'shoulders of the public education network of the city of \\ Montes Claros - MG
}

Prevalencia de lesiones en los ombres de docentes de la red de ensino público de la ciudad de Montes Claros - MG

João Victor Cardozo Silva ${ }^{1 *}$, Ana Caroline Cardozo Silva², João Jefferson Carneiro Corrêa ${ }^{2}$, Eric Hudson Evangelista e Souza², Danielle Cardoso Ribeiro², João Pedro Gonçalves Nemesio da Silva², Saulo Daniel Mendes Cunha ${ }^{3}$.

\section{RESUMO}

Objetivo: Verificar a prevalência de lesões nos ombros de professores da rede pública da cidade de Montes Claros-MG. Métodos: Trata-se de um estudo descritivo, com abordagem quantitativa e de corte transversal composto por professores do ensino médio e fundamental de escolas públicas da cidade de Montes Claros-MG. Foram selecionadas quatro instituições públicas com maior número de docentes que totalizou 256 docentes. 0 instrumento utilizado na coleta de dados foi o Questionário Nórdico para avaliação dos sintomas osteomusculares. Para análise dos dados foi utilizado o programa Microsoft Exceß ${ }^{\circledR}$ versão 2016. Os dados foram analisados através da estatística descritiva, com determinação das médias e desvio-padrão. Resultados: Predominaram indivíduos do sexo feminino com média de idade de 42,20 anos e desvio padrão $\pm 8,78$, com carga horária média de 24,14 horas e desvio padrão $\pm 10,90$. O ombro foi a região mais acometida nos últimos 12 meses. Conclusão: Os resultados do presente estudo demonstram a necessidade de novas pesquisas em relação aos aspectos ergonômicos do trabalho docente, além da criação de politicas públicas que visem à prevenção de adoecimento e promoção do bem-estar dos professores.

Palavras Chave: Professores, lesões osteomusculares, ombros.

\begin{abstract}
Objective: To verify the prevalence of lesions on the shoulders of teachers of the public network of the city of Montes Claros-MG. Methods: This is a descriptive study with a quantitative and cross-sectional approach composed of high school and elementary school teachers from the city of Montes Claros, MG. Four public institutions in the city of Montes Claros were selected with the highest number of teachers with 256 teachers. The instrument used to collect data was the Nordic Questionnaire for evaluation of musculoskeletal symptoms. For statistical analysis of the data, a database was prepared using the Microsoft Excel® software version 2016. Data were analyzed through descriptive statistics, with determination of means and standard deviation. Results: Predominant female subjects with mean age of 42.20 years and standard deviation \pm 8.78 , with an average hourly load of 24.14 hours and standard deviation \pm 10.90 . The shoulder was the most affected region in the last 12 months. Conclusion: The results of the present study demonstrate the need for new research on the ergonomic aspects of teaching work, as well as the creation of public policies aimed at preventing illness and promoting the well-being of teachers.
\end{abstract}

Key words: Teachers, musculoskeletal injuries, shoulders.

1'Universidade Estadual de Montes Claros (UNIMONTES), Montes Claros-MG. *E-mail: joaovitor cardozo@hotmail.com 


\section{RESUMEN}

Objetivo: Verificar la prevalencia de lesiones en los hombros de profesores de la red pública de la ciudad de Montes Claros-MG. Métodos: Se trata de un estudio descriptivo, con abordaje cuantitativo y de corte transversal compuesta por profesores de la enseñanza media y fundamental de escuelas públicas de la ciudad de Montes Claros-MG. Se seleccionaron cuatro instituciones públicas de la ciudad de Montes Claros con mayor número de docentes con el número de 256 docentes. El instrumento utilizado en la recolección de datos fue el Cuestionario Nórdico para la evaluación de los síntomas osteomusculares. Para el análisis estadístico de los datos se elaboró una base de datos utilizando el programa Microsoft Excel® versión 2016. Los datos fueron analizados a través de la estadística descriptiva, con determinación de las medias y desviación estándar. Resultados: Predominaron individuos del sexo femenino con promedio de edad de 42,20 años y desviación estándar $\pm 8,78$, con carga horaria media de 24,14 horas y desviación estándar $\pm 10,90$. El hombro fue la región más acometida en los últimos 12 meses. Conclusión: Los resultados del presente estudio demuestran la necesidad de nuevas investigaciones en relación a los aspectos ergonómicos del trabajo docente, además de la creación de políticas públicas que apunte a la prevención de enfermarse y promover el bienestar de los profesores.

Palabras clave: Profesores, lesiones osteomusculares, hombros.

\section{INTRODUÇÃO}

A docência é uma das mais antigas ocupações. O docente, anteriormente visto como uma figura essencial para a sociedade, hoje é um trabalhador que luta pela valorização e o reconhecimento social (LEMOS JC, 2005).

Segundo Santos MN e Marques AC (2013) ensinar é, em geral, uma atividade altamente estressante, com repercussões evidentes na saúde física, mental e no próprio desempenho profissional dos professores, que além de ministrar aulas, realizam trabalhos administrativos, planejamentos, orientam os alunos e interagem com os pais de seus alunos. Além disso, tem que organizar atividades extracurriculares, participar de reuniões de coordenação, seminários, conselhos de classe; preencher relatórios relativos às dificuldades de aprendizagem e, muitas vezes, cuidar do patrimônio, materiais, recreios e locais de refeições fazem parte de sua rotina de trabalho.

Neste sentido, é necessário entender como o trabalho docente pode influenciar no aparecimento de lesões em professores, como a doença osteomuscular relacionada ao trabalho (DORT). DORT é uma síndrome que acomete pessoas que trabalham por um longo período de tempo, acarretando uma série de sintomas que podem estar relacionados com sensação de fadiga muscular, dor, formigamentos ou pontadas principalmente em membros superiores (VERONESI JR, 2008).

A DORT é uma alteração do sistema musculoesquelético por usar diversas vezes, de forma repetida ou pela postura inadequada devido à implantação de novas tecnologias que não estão adaptadas ao corpo humano (SAKATA RK e ISSY AM, 2008).

Estas patologias podem acometer sobremaneira, os professores, que apresentam atividades profissionais relacionadas a problemas motivados por condições inadequadas de trabalho, posturas inapropriadas e movimentos repetitivos de determinados segmentos corporais. Assim, a necessidade por maiores jornadas de trabalho, maior produtividade e exigências de qualidade, são fatores predisponentes ao aumento dos casos de lesões que surgem em razão deste processo (COELHO CT, et al., 2010).

No contexto de atuação dos professores, identificam-se movimentos críticos, posturas inadequadas e condições ergonômicas errôneas que podem ser fatores determinantes na formação de lesões, tais como: escrever no quadro negro por longo período de tempo, com elevação dos membros superiores (MMSS) acima da cabeça, digitar e corrigir trabalhos e provas, entre outras.

Esses fatores podem levar a um estresse biomecânico no ombro, predispondo as articulações e ligamentos envolvidos no ombro a possíveis lesões, e consequentemente a dor, desconforto e possibilidades de afastamento da atividade laboral (BRAVO GBG, 2017). 
Em seu artigo, Freitas JRS (2007) cita que os aspectos que têm sido relacionados aos quadros músculoesqueléticos englobam componentes do processo de trabalho, incluindo a ação de atividades repetitivas, monótonas e a intensidade do esforço físico-postural, condições da estrutura do trabalho, aspectos psicossociais e propriedades biológico-individuais.

Segundo Coelho CT, et al. (2010) no quadro das DORT, a lesão que acomete o manguito rotador caracterizase como a causa mais frequente de dor no ombro podendo acometer indivíduos de qualquer faixa etária, sendo potencializada com o envelhecimento e a ocupação laboral ou recreativa.

Estudos comprovam alta prevalência desses sintomas dolorosos em trabalhadores, e os professores estão entre os profissionais mais acometidos, alcançando 93,0\% dos investigados (FERNANDES MH, et al., 2009).

Muitos desses sintomas acarretam o afastamento do trabalho, por quadros álgicos intensos ou pelo desenvolvimento de doenças ocupacionais. De acordo com o Centro de Estudos da Saúde do Trabalhador (CESAT) de Salvador, BA, do total de investigados, 66\% dos professores atendidos entre os anos de 1991 a 2001 foram diagnosticados com doença ocupacional (PORTO LA, et al., 2004).

Diante disto, os sintomas osteomusculares têm sido uma preocupação dos pesquisadores, pois se trata de uma questão de saúde e trabalho, em virtude dos custos e o impacto na qualidade de vida dos trabalhadores (PENTEADO RZ e PEREIRA IMTB, 2007).

Este estudo teve como objetivo verificar a prevalência de lesões nos ombros de docentes da rede pública de ensino da cidade de Montes Claros-MG.

\section{METODOLOGIA}

Este estudo caracteriza-se como quantitativo descritivo e de corte transversal. A população desta pesquisa foi composta por 256 docentes atuantes na rede pública da cidade de Montes Claros - MG.

A composição da amostra foi determinada por conveniência e com base no número de docentes atuantes nas instituições de ensino. Foram selecionadas as quatro instituições públicas da cidade de Montes Claros-MG com maior número de docentes. O cálculo amostral com índice de confiança de $95 \%$ e erro amostral de $5 \%$ revelou um número de 256 docentes a serem pesquisados. Os dados referentes aos números de escolas e número de docentes foram fornecidos pela Superintendência Regional de Ensino da cidade de Montes ClarosMG. As visitas iniciais as escolas serviram para esclarecer a proposta de estudo e para assinatura do termo de consentimento que garantiu a concordância da instituição em participar.

Em um segundo momento os pesquisadores retornaram à instituição para aplicação do questionário Nordic Musculoskeletal Questionnaire traduzido e validado para a população brasileira por Pinheiro FA, et al. (2002). Este instrumento consiste em um conjunto de escolhas múltiplas ou binárias referentes à ocorrência de sintomas nas diversas regiões anatômicas nas quais são mais comuns.

Os critérios de inclusão foram: Professores de ambos os sexos, que tenham mais de cinco anos de atuação na área de docência; que não apresentaram diagnóstico de doenças ocupacionais crônicas; não apresentaram atestado médico por motivo de problemas relacionados ao trabalho nos últimos 6 (seis) meses a data de aplicação do questionário; não estavam em tratamento de lesões/doenças ocupacionais; e os que demonstraram interesse e voluntariedade em participar da pesquisa. Além de ter formação acadêmica em qualquer área de ensino e aceitar assinar o termo de consentimento livre e esclarecido. Foram critérios de exclusão da pesquisa professores com licença escolar, professores que se recusaram em participar da pesquisa; os e professores que não tiverem no mínimo cinco anos de atuação na área de docência; e o que não apresentaram o Termo de concordância livre e esclarecido (TCLE) assinado.

Participaram da pesquisa respondendo ao questionário $172(67.18 \%)$ professores. Em relação aos 84 (32.81\%) professores que não participaram da pesquisa, 8,91\% recusaram-se verbalmente, $5,46 \%$ estavam de licença médica no período da coleta de dados, $6,56 \%$ não foram encontrados nas escolas em nenhum momento pelo pesquisador, 8,32\% não devolveram os questionários e 3,56\% não assinaram o TCLE assinado. 
As coletas de dados foram iniciadas após a aprovação do projeto pelo Comitê de Ética em Pesquisa com Seres Humanos. Foi explicado para os participantes todos os motivos e a importância da realização da pesquisa e o caráter de voluntariado e anonimato do estudo. Foi informado também que qualquer um poderia desistir da pesquisa a qualquer momento, e que todos os dados produzidos seriam utilizados exclusivamente para fins científicos, sendo seu armazenamento e publicação de responsabilidade do pesquisador.

Para análise dos dados elaborou-se um banco de dados utilizando o programa Microsoft Exceß versão 2016. Os dados foram analisados através da estatística descritiva, com determinação das médias e desvio-padrão da idade, tempo de docência e carga horária dos professores.

O estudo em questão não apresentou nenhum risco ou desconforto relacionados à dor, tontura, dores musculares. Caso algum voluntário se sentisse incomodado ou desconfortável durante a aplicação do instrumento, o mesmo poderia solicitar o desligamento da pesquisa.

\section{RESULTADOS}

Entre os professores investigados 119 (69.18\%) eram do sexo feminino e 53 (30.81\%) do sexo masculino, sendo a média de idade dos participantes 42,20 anos (D.P. $\pm 8,78$ ) (Tabela 1). A maior incidência de professores do sexo feminino também foi encontrada nos estudos de Cardoso JP, et al. (2009) com (92,0\%), e no estudo de Carvalho AJFP e Alexandre NMC (2006) 99,4\%. Em coerência com os dados da presente pesquisa, Vedovato TG e Monteiro MI (2008) analisaram os docentes de escolas Estaduais em Campinas e São José do Rio Pardo, usando um questionário que apresentavam dados sócios demográficos, hábitos de vida, trabalho, aspecto de saúde e riscos ocupacionais, os quais tiveram, também, a predominância do sexo feminino com o percentual de $81,8 \%$.

Tabela 1 - Descrição da Amostra

\begin{tabular}{|c|c|c|c|}
\hline Variáveis & $\mathbf{N}$ & Média & Desvio Padrão \\
\hline Sexo & & & \\
\hline Masculino & 53 & & \\
\hline $\begin{array}{l}\text { Feminino } \\
\text { Idade }\end{array}$ & 119 & & \\
\hline Idade Máx. & 62 anos & 42,20 & $\pm 8,78$ \\
\hline $\begin{array}{l}\text { Idade Mín. } \\
\text { Tempo de docência }\end{array}$ & 25 anos & & \\
\hline Maior Tempo de docência & 36 anos & 15,65 & $\pm 7,30$ \\
\hline Menor Tempo de docência & 05 anos & & \\
\hline Carga Horária & & 24,14 & $\pm 10,90$ \\
\hline
\end{tabular}

Fonte: Dados coletados de docentes da Rede de Ensino Público da Cidade de Montes Claros - MG, 2019.

A predominância do sexo feminino é semelhante ao relatado por Delcor NS et al. (2004) e Reis EJFB et al. (2005) em pesquisas realizadas com professores, que destacam a grande presença das mulheres nessa profissão, fato que está relacionado ao papel cultural da mulher na sociedade de educar e cuidar dos filhos, sendo então, essa profissão considerada como uma extensão do trabalho doméstico.

A partir da segunda metade do século XX ocorreu a expansão do setor educacional no Brasil, em que foi preciso a inserção de muitos trabalhadores para o ensino. A docência, tal quanto à enfermagem, foi considerada na época, atividade característica das mulheres por envolver o cuidado dos outros. As mulheres foram chamadas para ocupar os ofícios de educadoras, tendo em vista o trabalho na escola como uma continuação das tarefas exigidas no âmbito doméstico, aparecendo a imagem da "mãe educadora". Os baixos salários dos educadores também fazem com que as mulheres preencham esses trabalhos, a fim de aumentar a renda familiar (DELCOR NS, et al., 2004).

O tempo médio na função da docência foi de 15,65 (D.P. \pm 7,30) (Tabela 1). Sendo o maior tempo de docência 36 anos e o menor 05 anos. Comparando se com a presente pesquisa, Carvalho AJFP e Alexandre NMC (2006) constataram que os docentes da sua amostra, atuam na profissão a um tempo médio de 16,4 anos (DP $\pm 6,82$ ). 
Em consonância com esta pesquisa, Cardoso JP et al. (2009) descreveram em seu trabalho a prevalência de dor musculoesquelética, segundo variáveis sócias demográficas e ocupacionais de professores do ensino básico e constataram que o tempo médio entre os pesquisados foi de 14,4 anos na função de professor.

A média da carga horária semanal dos professores entrevistados foi de 24,14 horas semanais (D.P. $\pm 10,90$ ) (Tabela 1). A maioria dos professores entrevistados trabalha em uma escola. No estudo de Vedovato TG e Monteiro MI (2008), a carga horária média semanal dos professores em apenas uma escola foi um pouco maior, 26,2 horas (DP 10,1), e a jornada média de trabalho semanal em mais de uma escola era de 35,1 horas (DP 12,2). Vedovato TG e Monteiro MI (2008) sugerem que a carga horária máxima dos professores pode ser por trabalharem em mais de uma escola, pela necessidade de complementação da renda familiar, devido à baixa remuneração desses profissionais, o que eleva o período de exposição a diversos fatores de risco.

Do total de investigados, 103 (59.88\%) pessoas responderam SIM, e 69 (40.11\%) pessoas responderam NÃO, para prevalência de dor, formigamento e dormência nos últimos 12 meses, na região do ombro (Gráfico 1). Tais resultados tiveram algumas semelhanças com o estudo de Carvalho AJFP e Alexandre NMC (2006), como um alto percentual de dor em ombros, confirmando que são as regiões mais sobrecarregadas nos docentes e, logo, merecem atenção aos processos de cuidados e prevenção.

Gráfico 1 - Prevalência de dor, formigamento e dormência na região do ombro.

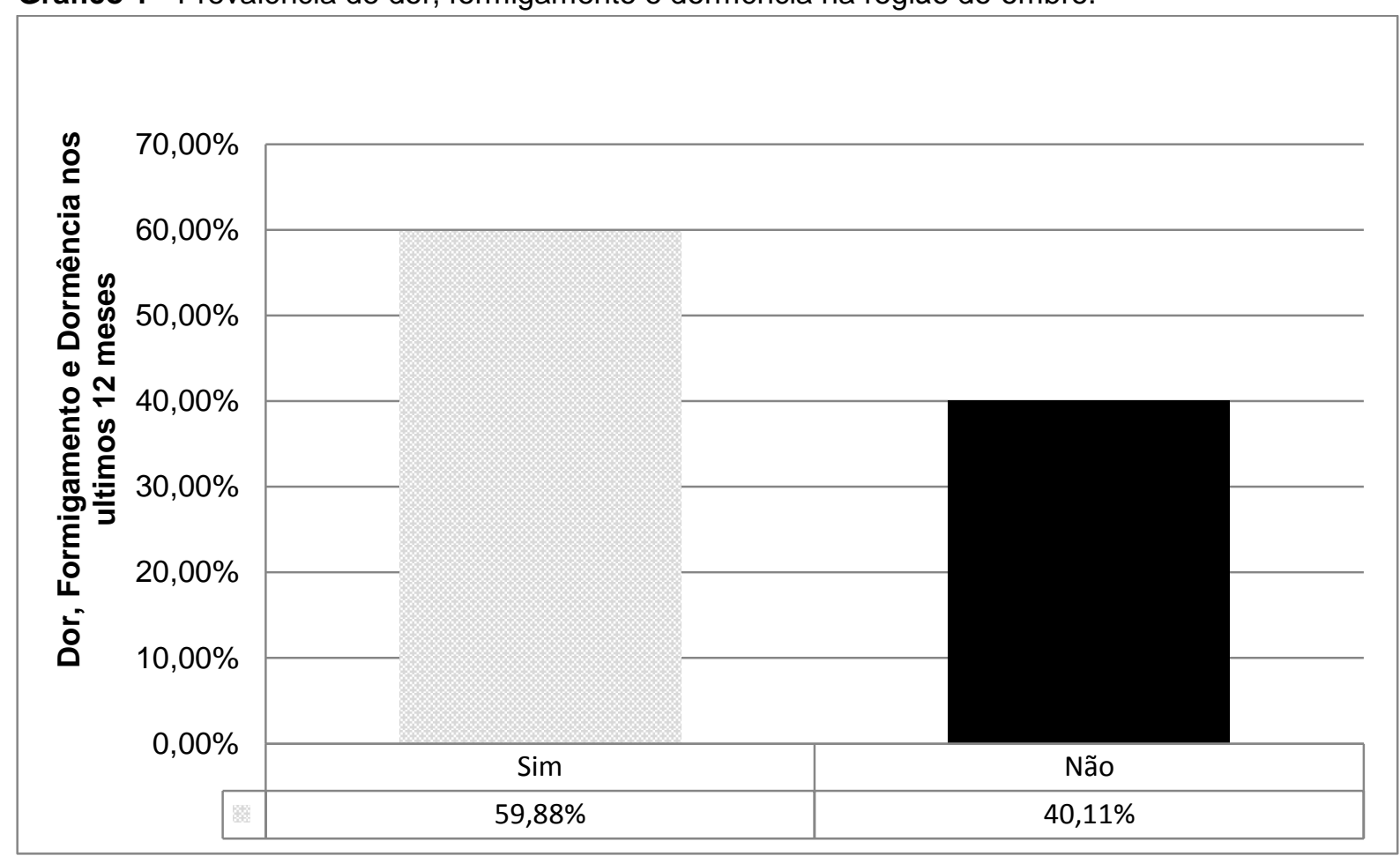

Fonte: Dados da pesquisa, 2019.

Dutra D, et al. (2005) verificaram em seus estudos que a maioria dos professores entrevistados $(76 \%)$ revelou dor em ombro, uma vez que ficam com o membro superior elevado em mais de $90^{\circ}$, levando a compressão dos tecidos moles, tais como a bursa subacromial, o tendão do supraespinhoso e o da cabeça longa do bíceps braquial. Valente MAS, et al. (2012) salientam que, ao se trabalhar com os braços acima de $90^{\circ}$, ocorre contração estática e ausência de vascularização nos músculos do manguito rotador, ocorrendo, então, compressão do tendão do músculo supraespinhoso, o que pode levar a dores subsequentes das tendinites e bursite.

Dessas 103 (59.88\%) pessoas que responderam Sim para dor, formigamento e dormência na região do ombro, 27 (26.21\%) pessoas, nos últimos 12 meses, foram impedidas de realizar trabalhos normais como, por exemplo, trabalhar, realizar atividades domésticas e de lazer por causa desses problemas relacionados ao ombro (Gráfico 2). 
Gráfico 2 - Afastamento na atividade laboral.

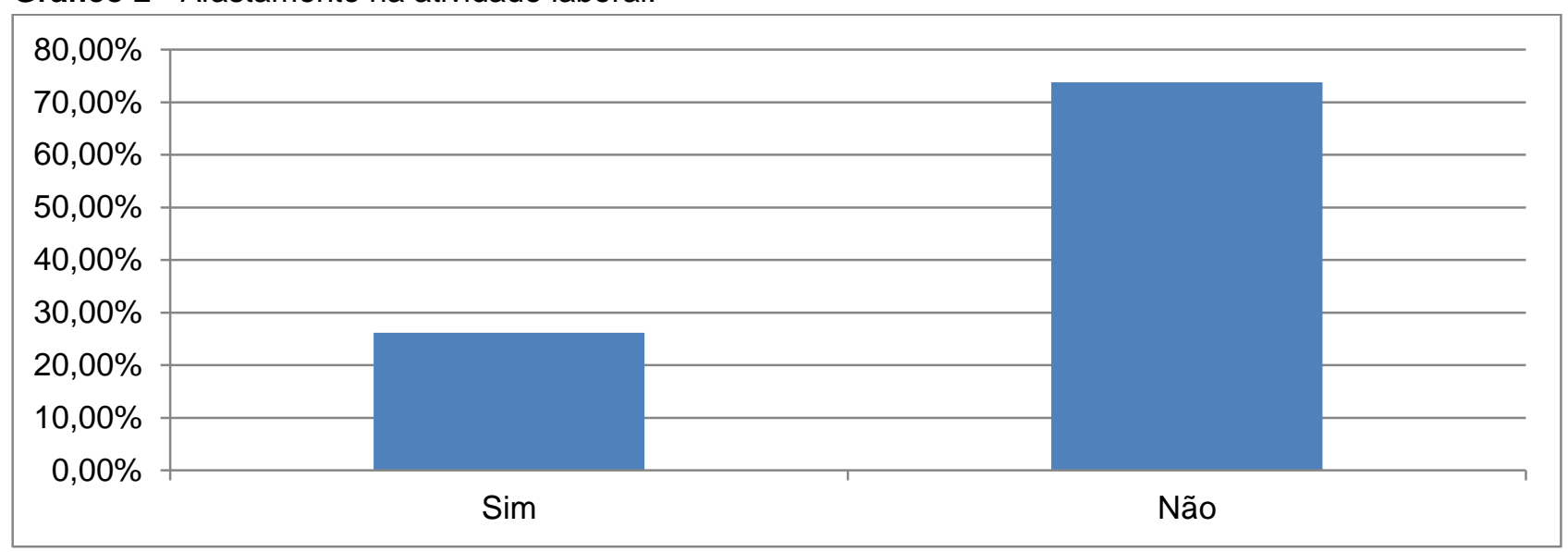

Fonte: Dados da pesquisa, 2019.

Schuster M e Schroeder TMR (2017) em sua pesquisa abordam o adoecimento de professores com o objetivo de identificar as doenças que têm causado seus afastamentos do trabalho na Rede Municipal de Educação da cidade de Cascavel, no Paraná. Foram realizados levantamentos em atestados médicos cadastrados no ano de 2014 junto à Divisão de Medicina e Segurança do trabalho da Prefeitura Municipal desta cidade, para uma análise quanti-qualitativa. A análise dos atestados cadastrados evidenciou que dos 394 atestados averiguados $96 \%$ eram de professoras, com idade média de 41,83 anos. O terceiro maior motivo de afastamentos apresentado pelos professores de Cascavel foram distúrbios musculoesqueléticos (13,95\%), com 55 atestados cadastrados. Desses, seis por Lesões de Ombro.

Diferindo dos achados do presente estudo em relação aos professores (as) que ficarem impedidos (as) de realizar trabalhos normais, Dutra D, et al. (2005) relataram em seu estudo que $10 \%$ dos professores com dor se afastaram da função por no mínimo um episódio, contra $78 \%$ dos entrevistados, que embora com a presença da dor permaneciam trabalhando, apesar de apresentar desconforto.

Apesar de 27 (26.21\%) pessoas ficarem impedidas de realizar suas atividades de vida diária, 35 (33,98\%) dos pesquisados afirmou ter procurado um profissional da área da saúde devido a algum sintoma musculoesquelético nos últimos 12 meses (Gráfico 3), o que confirma os dados da literatura de Panzeri AJF (2004). Tal fato pode ser justificado por um aumento das incapacidades ocupacionais, prejudicando assim o desempenho dos docentes no desempenho de seu trabalho, o que resulta na redução de sua produtividade.

Gráfico 3 - Consulta a algum profissional da saúde, após identificar a lesão.

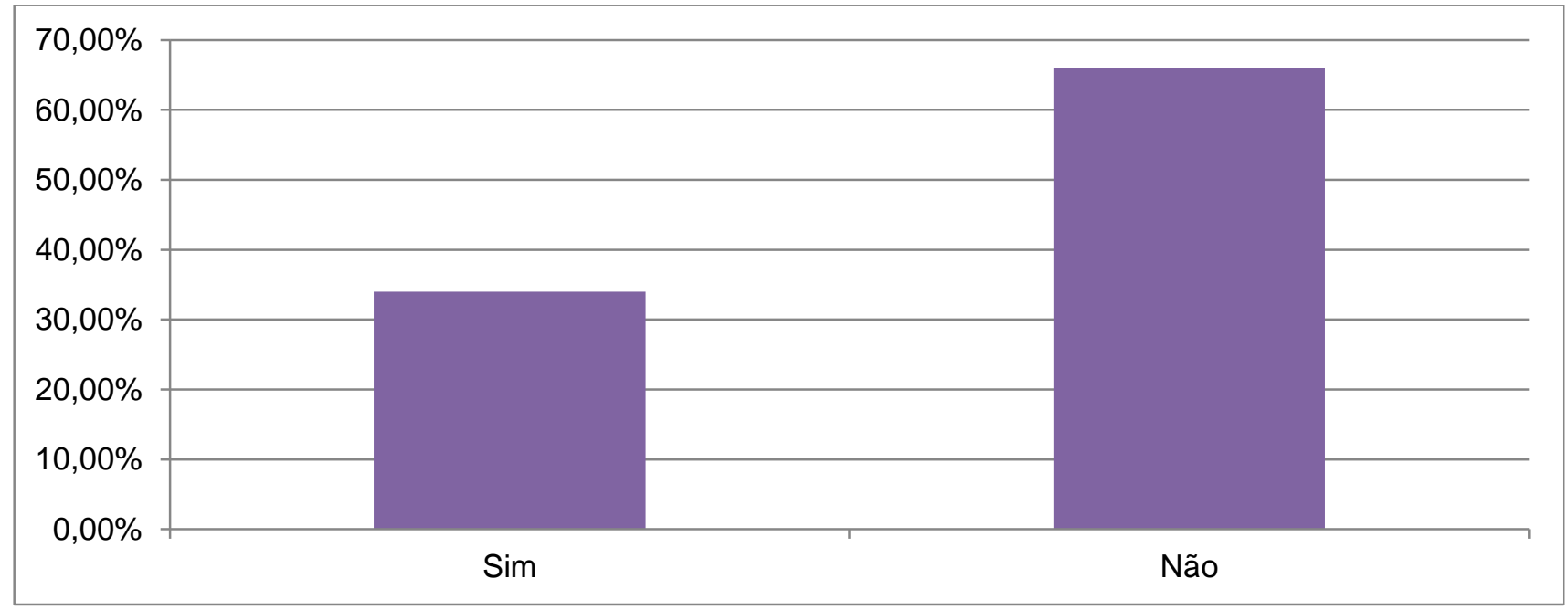

Fonte: Dados da pesquisa, 2019. 
No estudo de Melo EMN, et al. (2010), do total de investigados, 96,3\% responderam que a presença da sintomatologia osteomuscular nos últimos 12 meses impediu a realização das atividades de vida diária, tendo $96 \%$ dos participantes relatado ter realizado consulta a algum profissional da saúde devido ao referido problema.

Em relação à dor, formigamento ou dormência, 43 (41.74\%) dos entrevistados afirmaram que tiveram algum problema na região do ombro nos últimos sete dias (Gráfico 4). Panzeri AJF (2004), em sua pesquisa, obteve uma percentagem de $29,9 \%$ de queixas no ombro nos últimos sete dias, no seu estudo realizado com 157 professores efetivos da rede estadual e municipal de ensino da cidade de São João da Boa Vista-SP.

Melo EMN, et al. (2010) analisou a última questão do Questionário Nórdico, em que 95\% dos entrevistados afirmaram que tiveram algum problema nas regiões do corpo nos últimos 7 dias, no seu estudo realizado com 45 professores em 6 escolas da Rede Municipal da cidade de Catalão-GO. Dentre as regiões que apresentaram maior número de queixas, destacamos o ombro $18 \%$.

Gráfico 4 - Dor, formigamento ou dormência nos ombros, nos últimos 7 dias.

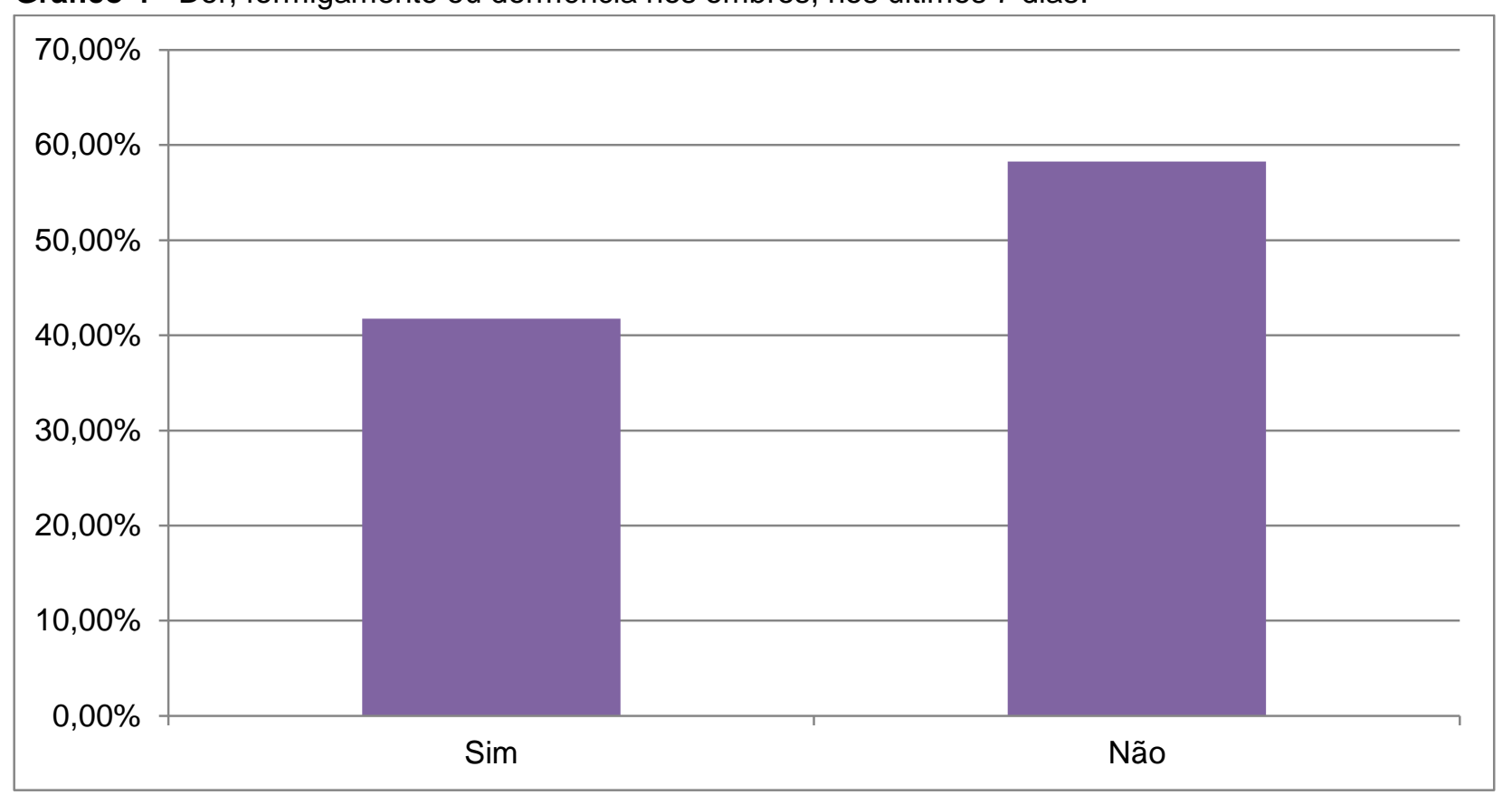

Fonte: Dados da pesquisa, 2019.

Os resultados do presente estudo demonstram a necessidade de novas pesquisas em relação aos aspectos ergonômicos e organizacionais do trabalho docente. Este estudo não representa o percentual das escolas de Montes Claros-MG, pois existem diferentes realidades entre as escolas públicas. Para que isso não venha se tornar um problema de saúde coletiva dessa classe de trabalhador, é fundamental que seja destacado e levado ao conhecimento das autoridades nacionais a fim de contribuir para adoção de politicas públicas que visem à prevenção de adoecimento e promovam bem-estar dessa categorial profissional.

\section{CONCLUSÃO}

O adoecimento do docente é uma questão que destaca a urgência da valorização profissional, do compromisso político em assegurar melhores condições de trabalho e de salários justos para que os docentes possam assumir adequadamente suas responsabilidades sociais na função de lecionar. A fim de que isso se efetive, é importante entender, afirmar e vivenciar o bem-estar na docência. Os achados deste estudo destacam a hipótese de que as características dessa atividade laboral trazem efeitos negativos para a saúde dos trabalhadores. Portanto, pela relevância deste estudo, este não deve se encerrar aqui, mas contribuir com novas pesquisas na área. 


\section{REFERÊNCIAS}

1. BRAVO GBG. Aspectos ergonômicos na utilização do quadro negro para os docentes da UTFPR. Monografia de especialização. Universidade Tecnológica Federal do Paraná, Curso de especialização em engenharia de segurança do trabalho, Londrina, Paraná, 2017; 50 p.

2. CARVALHO AJFP, ALEXANDRE NMC. Sintomas osteomusculares em professores do ensino fundamental. Revista Brasileira de Fisioterapia, 2006; 10(1): 35-41.

3. CARDOSO, JP et al. Prevalência de dor musculoesquelética em professores. Rev. bras. Epidemiol, 2009; 12(4): 604614.

4. COELHO CT, et al. Prevalência da síndrome do ombro doloroso (SOD) e sua influência na qualidade de vida em professores de uma instituição privada de nível superior na cidade de Lauro de Freitas. Revista Baiana de Saúde Pública, 2009; 34(1):19-29.

5. DELCOR NS, et al. Condições de trabalho e saúde dos professores da rede particular de ensino de Vitória da Conquista, Bahia, Brasil. Cadernos de Saúde Pública, 2004; 20:187-196.

6. DUTRA D, et al. Prevalência de algias nos ombros em professores da rede municipal de ensino fundamental de Umuarama - PR no ano de 2004. Arq. Ciênc. Saúde Unipar, 2005; 9(2): 79-84.

7. FREITAS JRS. Os distúrbios osteomusculares relacionados ao trabalho em profissionais de enfermagem de um hospital universitário. Dissertação (mestrado) - Fundação Universidade Federal do Rio Grande, Rio Grande, $2007 ; 73$ p.

8. FERNANDES MH, et al. Fatores Associados à Prevalência de Sintomas Osteomusculares em Professores. Revista de Salud Pública, 2009;11(2) 256-257.

9. LEMOS JC. Carga psíquica no trabalho e processos de saúde em professores universitários. Tese (Doutorado em Engenharia de Produção e Sistemas) - Universidade Federal de Santa Catarina. Florianópolis, 2005;147 p.

10. MELO EMN, et al. Prevalência de lesões osteomusculares em professores do ensino fundamental. Revista Eletrônica "Saúde CESUC" - Centro de Ensino Superior de Catalão, 2010; 1.

11. PINHEIRO FA, et al. Validação do Questionário Nórdico de Sintomas Osteomusculares como medida de morbidade. Rev. Saúde Pública, 2002; 36(3): 307-312.

12. PANZERI AJF. Sintomas osteomusculares e qualidade de vida em professores do ensino fundamental. Dissertação (mestrado) - Universidade estadual de Campinas, São Paulo, 2004; 146 p.

13. PENTEADO RZ, PEREIRA IMTB. Qualidade de vida e saúde vocal de professores. Rev. Saúde Pública, 2007; 41(2): 236-243.

14. PORTO LA, et al. Doenças ocupacionais em professores atendidos pelo Centro de Estudos da Saúde do Trabalhador (CESAT). Rev baiana saúde pública, 2004, 28(1): 33-49.

15. REIS EJFB, et al. Trabalho e distúrbios psíquicos em professores da rede municipal de Vitória da Conquista, Bahia, Brasil. Cad Saúde Pública. 2005; 21(5):1480-90.

16. ROCHA VM, FERNANDES MH. Qualidade de Vida de Professores do Ensino Fundamental: uma Perspectiva para a Promoção da Saúde do Trabalhador. Jornal Brasileiro de Psiquiatria, 2008; 57(1): 23-27.

17. SAKATA RK, ISSY AM. Dor. 2. ed, Manole, 2008.

18. SANTOS MN, MARQUES AC. Condições de saúde, estilo de vida e características de trabalho de professores de uma cidade do sul do Brasil. Ciência \& Saúde Coletiva, 2013; 8: 837-846.

19. SOUZA NA, LEITE MP. Condições de trabalho e suas repercussões na saúde dos professores da educação básica no Brasil. Educ Soc. 2011; 32(117):1115-21.

20. SCHUSTER M, SCHROEDER TMR. Estresse, dor e lesões musculoesqueléticas em professores de cascavel - PR. Revista educere et educare, 2017; 12(242).

21. VERONESI JR. Fisioterapia do Trabalho: Cuidando da Saúde Funcional do Trabalhador.1. ed. Andreolli: [S.n.], 2008.

22. VALENTE MAS, et al. Influência da elevação isométrica em abdução de ombro acima de $90^{\circ}$ em lesões osteomusculares relacionadas com atividade laboral. (Monografia) - Pós-graduação Latu sensu em Ergonomia. Faculdade Ávila, Goiânia, 2012; $12 \mathrm{p}$.

23. VEDOVATO TG, MONTEIRO MI. Perfil sociodemográfico e condições de saúde e trabalho dos professores de nove escolas estaduais paulistas. Rev. esc. enferm. USP, 2008; 42(2):291-297. 